\title{
Doppler Radar Analysis of a Tornadic Miniature Supercell during the Landfall of Typhoon Mujjgae (2015) in South China
}

\author{
Kun Zhao, Mingunn Wang, Ming Xue, Peiling Fu, Zhonglin Yang, Xiaomin Chen, \\ Yi Zhang, Wen-Chau Lee, Fuging Zhang, QIng Lin, and Zhaohu LI
}

$\mathrm{T}$ ropical cyclone (TC)-spawned tornadoes often occur in the outer rainbands located in the rightfront or the northeast quadrant relative to the TC track within a 200-400-km annulus (Edwards 2012; Schultz and Cecil 2009). These tornadoes often reside in miniature supercells in an environment characterized by a high low-level moisture content, a low lifting condensation level, a small surface dewpoint depression, small or moderate convective available potential energy (CAPE), and enhanced low-level shear due to increased surface friction from water to land, as well as boundary layer convergence (Green et al. 2011). Generally, these

AfFiliations: ZhaO, WANG, Fu, YANG, Chen, AND Y. ZhanG-Key Lab of Mesoscale Severe Weather/Ministry of Education of China, and School of Atmospheric Sciences, Nanjing University, Nanjing, China, and State Key Laboratory of Severe Weather and Joint Center for Atmospheric Radar Research of CMA/NJU, Chinese Academy of Meteorological Sciences, Beijing, China; XUE-Key Lab of Mesoscale Severe Weather/Ministry of Education of China, and School of Atmospheric Sciences, Nanjing University, Nanjing, China, and Center for Analysis and Prediction of Storms and School of Meteorology, University of Oklahoma, Norman, Oklahoma; LeEEarth Observing Laboratory, National Center for Atmospheric Research,* Boulder, Colorado; F. ZHANG—Department of Meteorology and Atmospheric Science, and Center for Advanced Data Assimilation and Predictability Techniques, The Pennsylvania State University, University Park, Pennsylvania; LIN AND LI-Guangdong Meteorological Observatory, and Foshan Tornado Research Center, Guangdong Meteorological Service, Guangzhou, China * The National Center for Atmospheric Research is sponsored by the National Science Foundation

CORRESPONDING AUTHOR: Dr. Kun Zhao, zhaokun@nju.edu.cn

DOI:10.II75/BAMS-D-15-0030I.I

(C2017 American Meteorological Society

For information regarding reuse of this content and general

copyright information, consult the AMS Copyright Policy. miniature supercells exhibit a hook echo/appendages and tornado vortex signatures (TVSs) in Doppler radar observations (McCaul 1987; McCaul et al. 2004). Rare dual-Doppler observations (e.g., Eastin et al. 2009; Lee et al. 2008) have provided snapshots of the kinematic and dynamic structures of miniature supercells in the United States in the past. In Asia, TC-spawned tornadoes and their parent miniature supercells have been observed by single Doppler radars in Japan and China (e.g., Saito et al. 1992; Suzuki et al. 2000; Zheng et al. 2015), revealing similar radar signatures as in their U.S. counterparts. In China, the average annual number of tornadoes over the past half century is less than 100, and the number of EF3 (on the enhanced Fujita scale) intensity or higher tornadoes is $\sim 20$ (Fan and Yu 2015). Typhoon rainband-spawned tornadoes occurred about once per year in China (Zheng et al. 2015). Nevertheless, the evolution, three-dimensional structures, and other dynamic characteristics of miniature supercells in Asia have yet to be documented through dual-Doppler wind analysis.

Typhoon Mujigae (2015) was categorized as a supertyphoon ( $>51 \mathrm{~m} \mathrm{~s}^{-1}$; Yu et al. 2013) by the Chinese Meteorological Administration (CMA) near the time of landfall in Guangdong province at 1400 LST 4 October 2015. The maximum sustained surface wind was $\sim 52 \mathrm{~m} \mathrm{~s}^{-1}$ and the minimum central sea level pressure (MSLP) was $935 \mathrm{hPa}$ (Fig. 1). Mujigae resulted in 19 deaths and $\$ 3.45$ billion in losses in China; thus, the name Mujigae has been retired by the World Meteorological Organization. After its landfall, Mujigae produced two confirmed tornadoes and one waterspout in three separate miniature supercells in the two outermost rainbands (Fig. 1). The strongest

Publisher's Note: This article was revised on 16 October 2017 to correct the reference for Suzuki et al. 2000. 


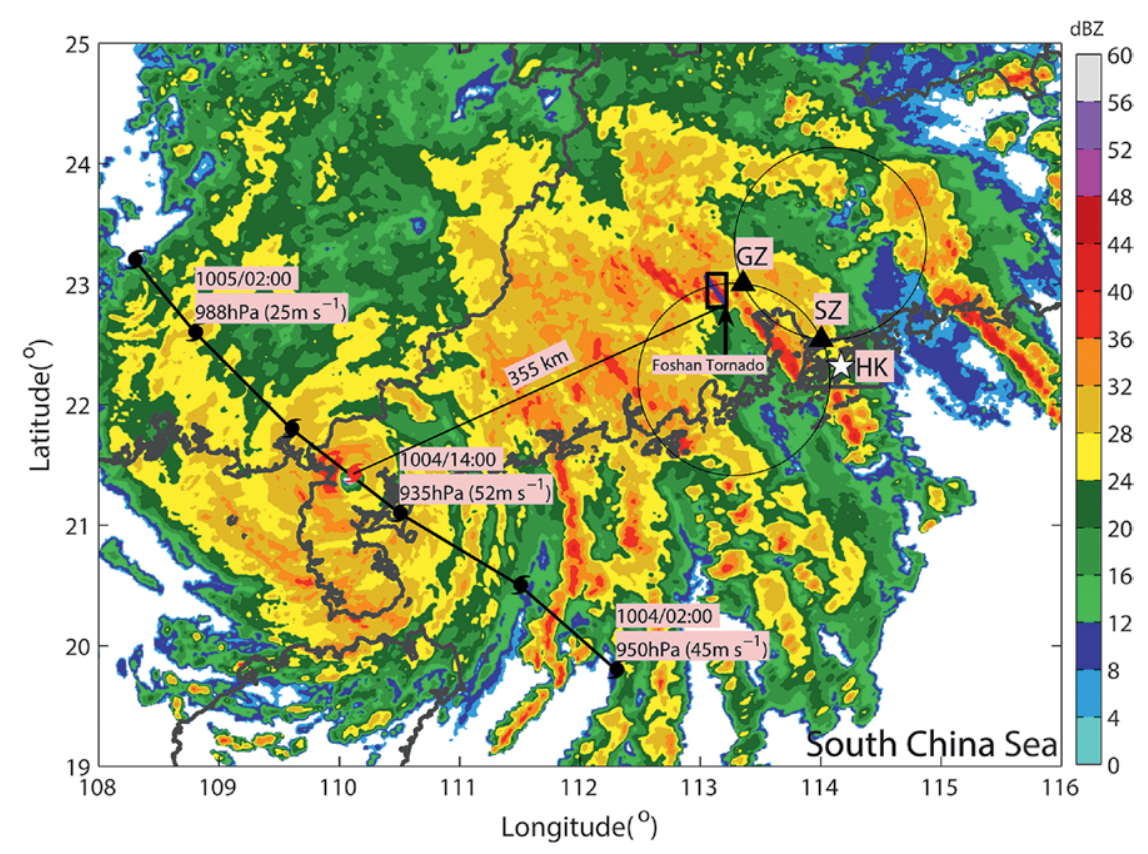

Fig. I. The radar sites (solid triangles) at Guangzhou (GZ) and Shenzhen (SZ) and the sounding station (white pentagram) at Hong Kong (HK) are overlaid on the composite reflectivity of Tropical Cyclone Mujigae at I500 LST 4 Oct 20I5. Two circles indicate the dual-Doppler radar analysis domain. The TC track is shown by the black line segments, with TC symbols representing the TC center every $6 \mathrm{~h}$, and the black rectangle outlines the region where Foshan tornado occurred. The tornado track is indicated by the thick blue line segment. tornado, spawned in the third rainband from the center, was located $\sim 350 \mathrm{~km}$ northeast (pole-relative quadrant) of Mujigae's center and was rated EF3, causing 4 deaths, up to 80 injuries, and about $\$ 29$ million in damage in the city of Foshan. These outer rainbands were accompanied by active lightning over the ocean, but lightning activity diminished over land. There were several weak lightning flashes recorded near the Foshan tornadic minisupercell, but no hail was reported. No tornado warnings were issued, as CMA is currently in the process of establishing a formal tornado warning protocol in China (Xue et al. 2016). Doppler radar data were collected from Guangzhou (GZ) and Shenzhen (SZ) over a 90-min period while the target miniature supercell was located in the western dual-Doppler lobe (Fig. 1). The purpose of this study is to document, for the first time, the time evolution of the strongest TC-spawned tornado ever observed by modern instruments in China, as well as the evolution and structure of the parent mesocyclone and miniature supercell. The paper also compares and contrasts the characteristics of the parent mesocyclone of this TC-spawned tornado with its U.S. counterparts using the Doppler radar data from the closer GZ radar, while coarser-resolution dual-Doppler wind analyses are used to examine the intensification mechanisms of the mesocyclone via vorticity budget analyses.

ENVIRONMENTAL CONDITIONS AND DAMAGE SURVEY. The environmental conditions of the TC miniature supercell are shown in Fig. 2, based on a rawinsonde released outside the tornadic rainband at the Hong Kong International Airport at 0600 UTC (1400 LST), which is about $100 \mathrm{~km}$ to the southeast and an hour before the miniature supercell became tornadic. The key buoyancy and shear parameters (Fig. 2), including a moderate CAPE $\left(\sim 1284 \mathrm{~J} \mathrm{~kg}^{-1}\right)$, a low lifting condensation level $(\sim 389 \mathrm{~m})$, a small surface dewpoint depression $(\sim 3 \mathrm{~K})$, a large veering low-level $(0-3 \mathrm{~km})$ vertical wind shear $\left(\sim 22.3 \mathrm{~m} \mathrm{~s}^{-1}\right)$, and a large cell-relative helicity $\left(\sim 211 \mathrm{~m}^{2} \mathrm{~s}^{-2}\right)$, meet the "high threat" category of a TC-spawned tornado in the United States (McCaul 1991; Schneider and Sharp 2007).

Damage surveys were conducted jointly by CMA, Nanjing University, and Peking University. Aerial photography along the damage path (Fig. 3) was taken by an unmanned aerial vehicle operated by the Foshan Meteorological Bureau during 8-10 October. Ground damage surveys were also conducted to mark the directions of fallen walls, trees, and power poles. The wind speed estimates of the matched degree of damage (DOD) were assigned the expected values (EXP in McDonald and Mehta 2006) for all of the damage indicators (DIs) (Meng and Yao 2014). Visual evidence (e.g., photographs and videos) of the tornado was also collected from a variety of sources (e.g., news websites and a variety of online social media), which provided photographic evidence of the tornado. The most severe damage was rated EF3, as recommended by the Wind Science and Energy Center (McDonald and Mehta 2006).

The tornado's damage track was about $30 \mathrm{~km}$ long (white line in Fig. 3c). The wind damage first appeared 

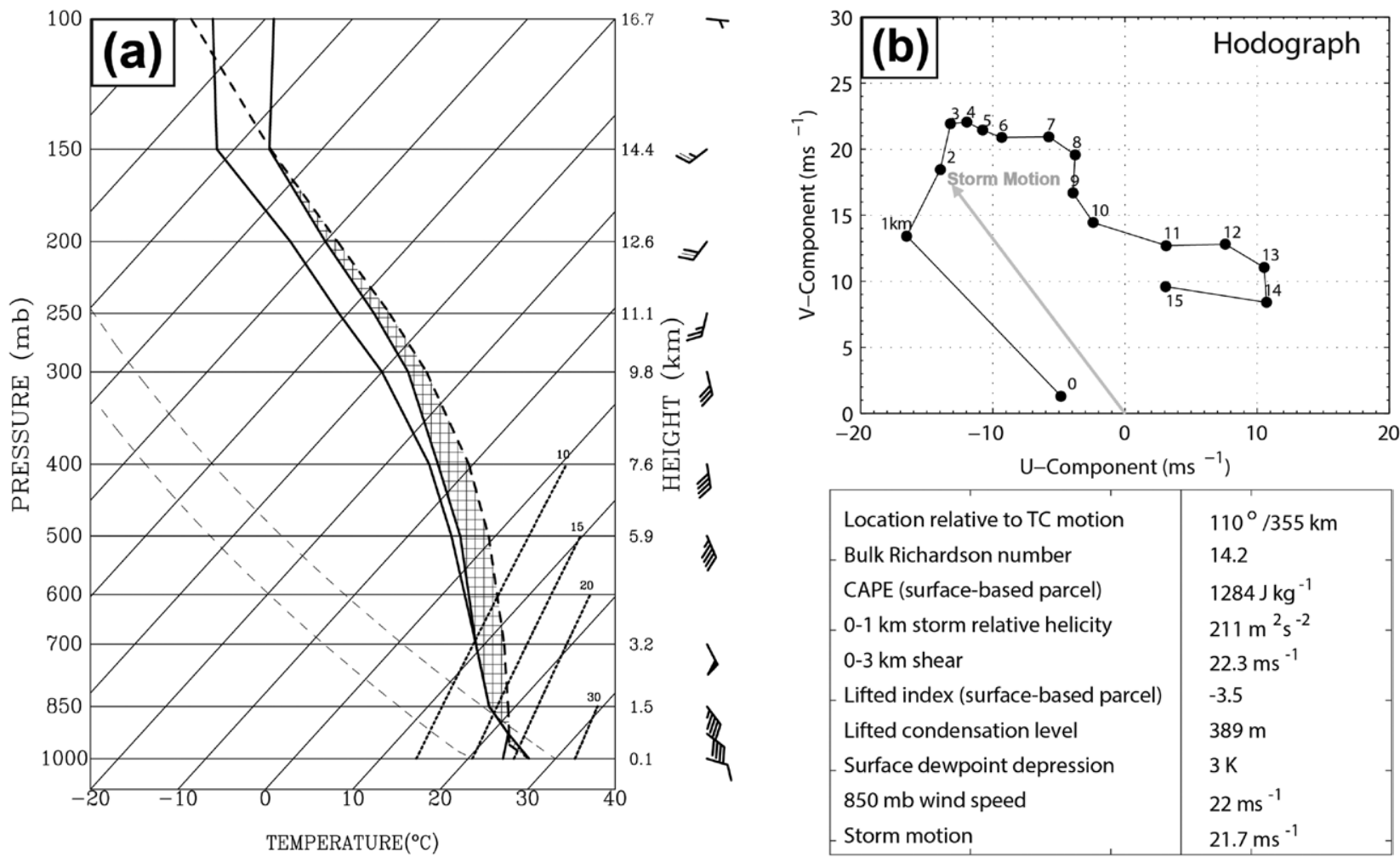

\begin{tabular}{|l|l|}
\hline Location relative to TC motion & $110^{\circ} / 355 \mathrm{~km}$ \\
- Bulk Richardson number & 14.2 \\
CAPE (surface-based parcel) & $1284 \mathrm{~J} \mathrm{~kg}^{-1}$ \\
$0-1 \mathrm{~km}$ storm relative helicity & $211 \mathrm{~m}^{2} \mathrm{~s}^{-2}$ \\
$0-3 \mathrm{~km}$ shear & $22.3 \mathrm{~ms}^{-1}$ \\
Lifted index (surface-based parcel) & -3.5 \\
Lifted condensation level & $389 \mathrm{~m}^{-1}$ \\
Surface dewpoint depression & $3 \mathrm{~K}$ \\
$850 \mathrm{mb}$ wind speed & $22 \mathrm{~ms}^{-1}$ \\
Storm motion & $21.7 \mathrm{~ms}^{-1}$ \\
\hline
\end{tabular}

FIG. 2. (a) Sounding profile and (b) hodograph at 1400 LST at the Hong Kong airport. Temperature and dewpoint profiles are represented by black solid lines, while a surface-based parcel path is shown by the black dashed line. The cross-hatched area represents a CAPE of $1284 \mathrm{~J} \mathrm{~kg}^{-1}$ for the lifted parcel. Winds (half barb $=5 \mathrm{~m} \mathrm{~s}^{-1}$; full barb $=10 \mathrm{~m} \mathrm{~s}^{-1}$ ) are also shown. The gray arrow in (b) represents the storm motion.

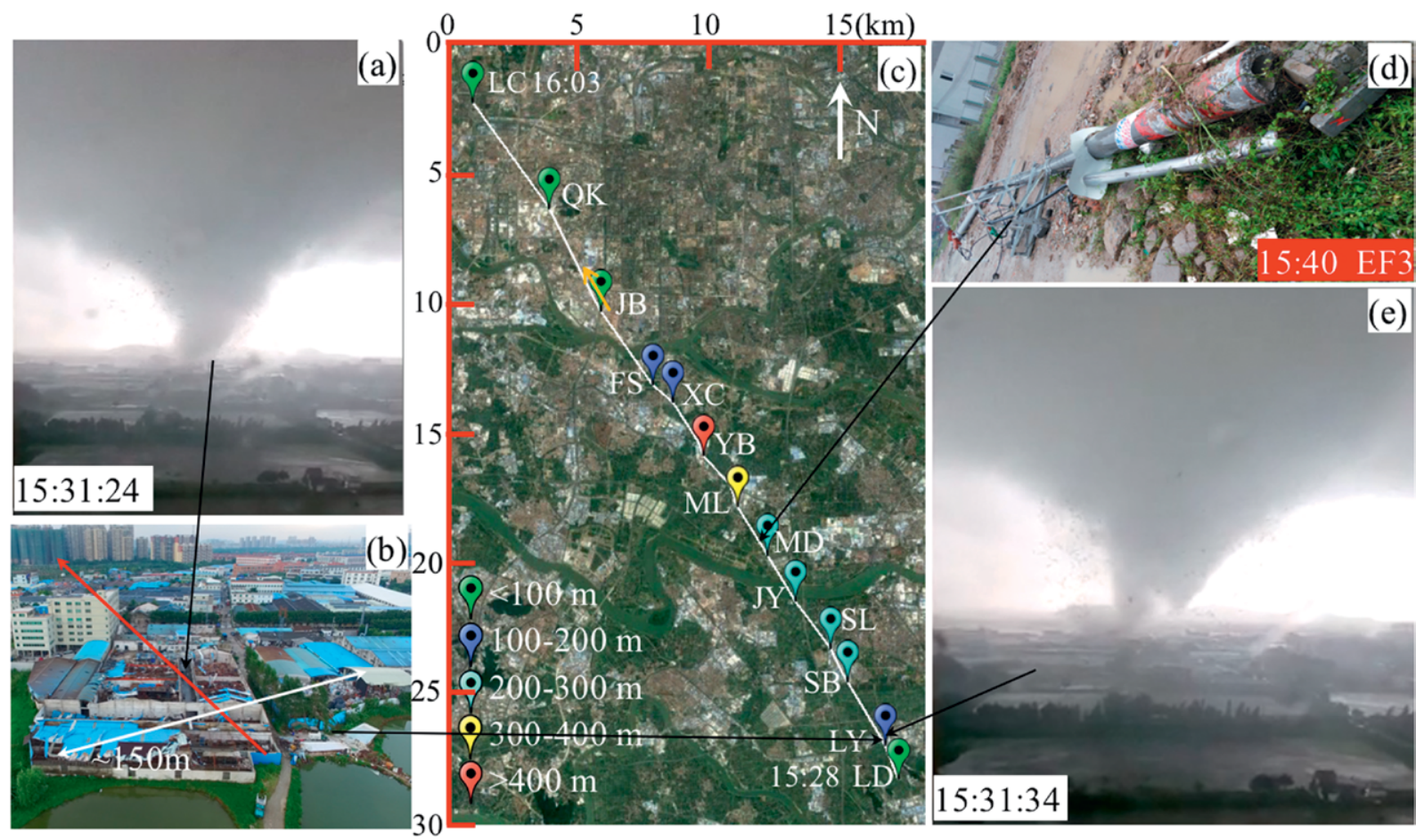

Fig. 3. (c) Damage survey of tornado path corresponding to the black rectangle in Fig. I. (a),(e) The tornado is viewed from the north and (b),(d) the associated damage. 
to the southeast of $\mathrm{LD}$, but without clear tornado damage patterns. The tornado's parent supercell moved north-northwest with a mean speed of $\sim 21 \mathrm{~m} \mathrm{~s}^{-1}$. At LY, a funnel cloud was captured by a cellphone camera at $1531: 24$ LST (Fig. 3a) by S. Huang (available on http://youku.com), suggesting that the tornado touched down between LD and LY. Then, 10 s later at 1531:34 LST, two funnels are apparent (Fig. 3e). Several factory buildings were completely demolished by the tornado between LD and LY, within a damage swath of $\sim 150 \mathrm{~m}$ (Fig. 3b). The DI of this steel-frame building with DOD 8 (total destruction of the building) was rated EF3. The tornado then hit SB at 1534 LST and damaged a larger group of steel-frame buildings (rated EF3; not shown). A concrete electrical transmission line pole was snapped (Fig. 3d) north of MD (rated EF3). The tornado maintained a high intensity while broadening. It reached its widest damage path $(\sim 500 \mathrm{~m})$ at $\mathrm{YB}$ with the same DOD as caused at LY and SB.

\section{RADAR SIGNATURES OF THE MINIATURE SUPERCELL, MESOCYCLONE, AND TOR-} NADO. Miniature supercell and mesocyclone. The damage track matched well with the hook-echo radar reflectivity signature and the accompanying Doppler velocity dipole where $V_{\max }$ and $V_{\min }$ are the

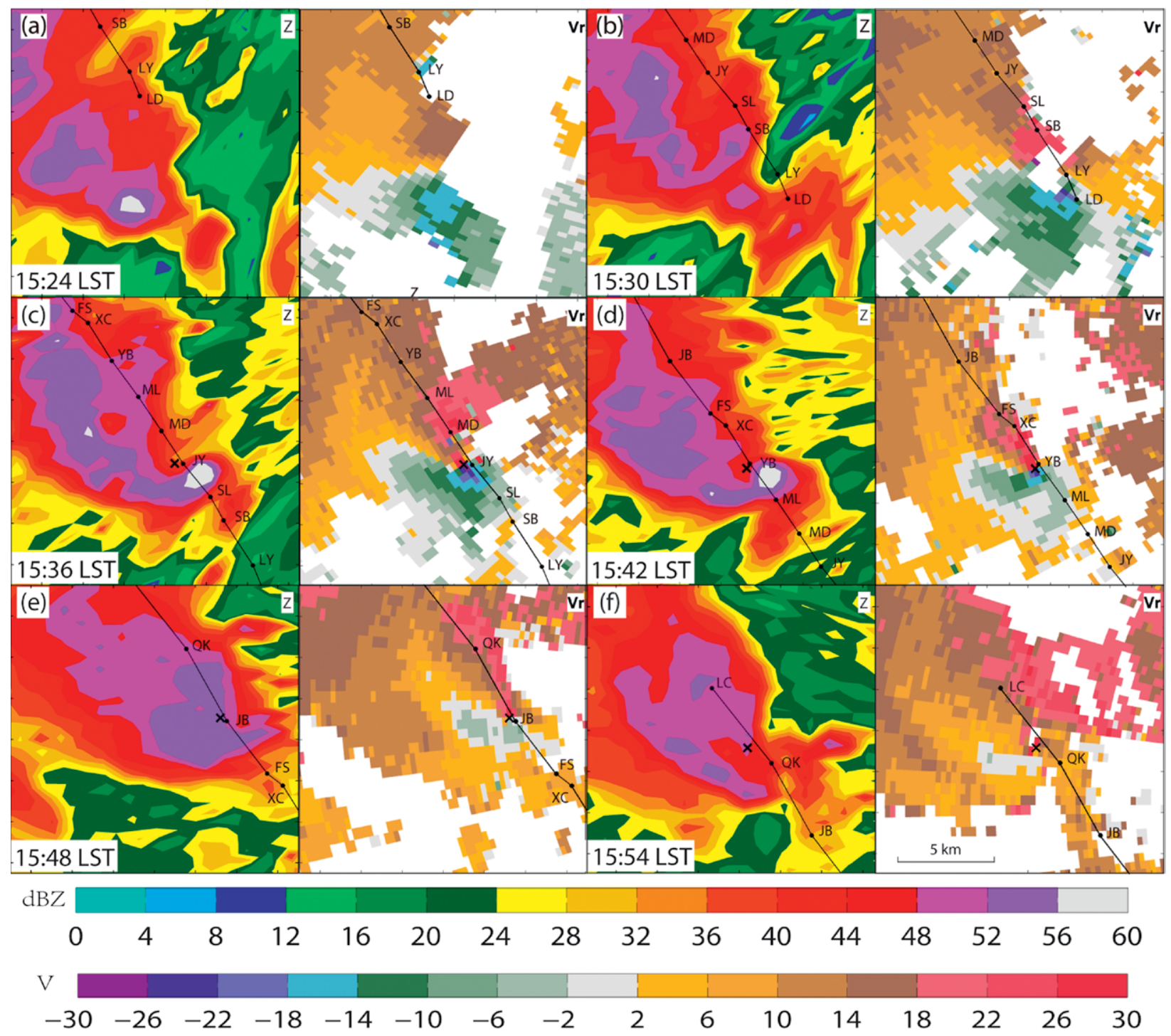

FIG. 4. Damage survey of tornado path (solid black line) from Fig. 3 and the TVS (black cross) superimposed on radar reflectivity $(\mathrm{dBZ})$ at $1.5^{\circ}$ elevation angle from the Guangzhou radar and ground-relative radial velocities $\left(\mathrm{m} \mathrm{s}^{-1}\right)$ from (a) 1524 to (f) 1554 LST at an interval of $6 \mathrm{~min}$. 

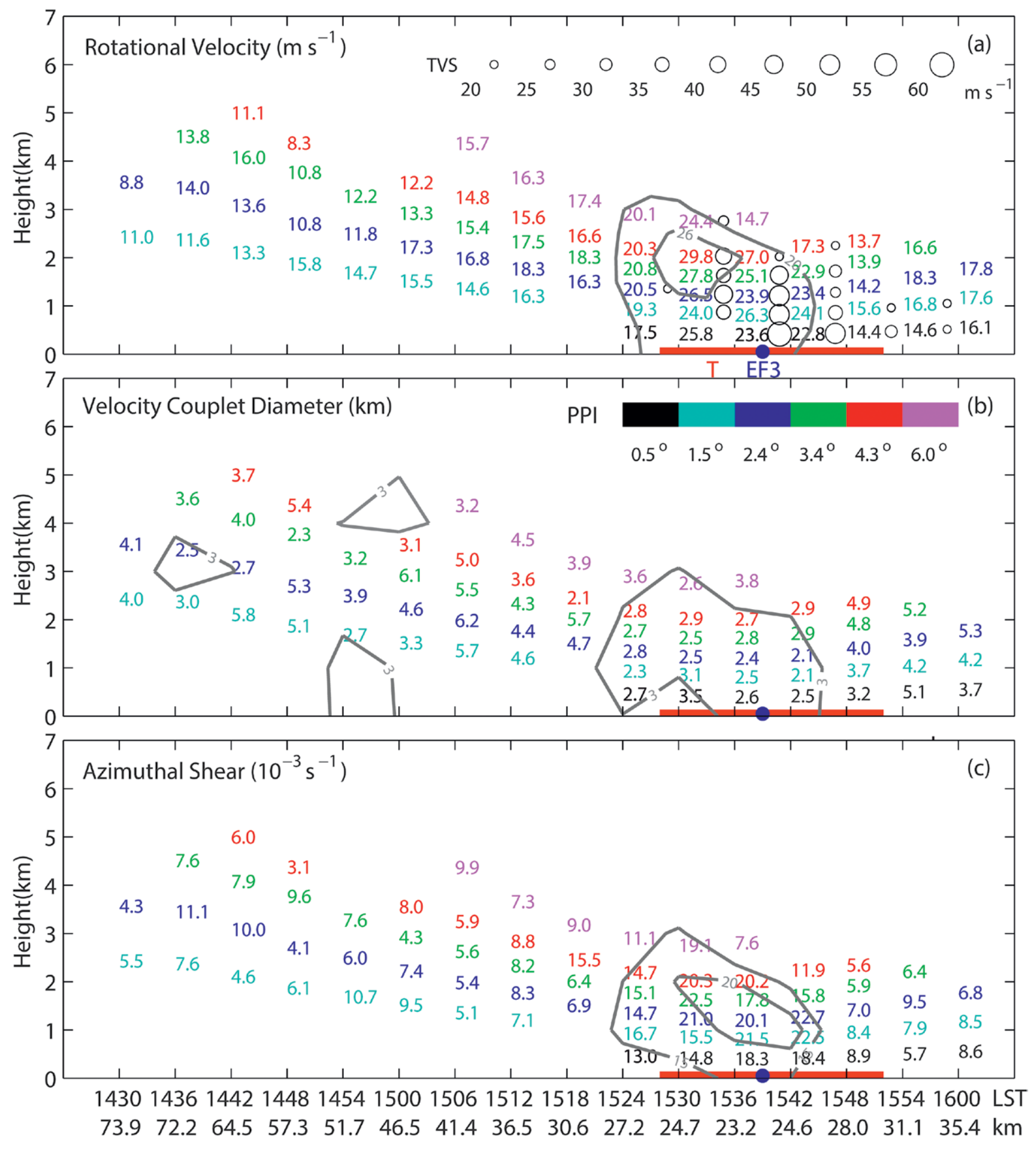

Fig. 5. Time-height profiles of (a) rotational velocity $\left(\mathrm{m} \mathrm{s}^{-1}\right.$; gray lines represent 20 and $26 \mathrm{~m} \mathrm{~s}^{-1}$ contours), (b) couplet diameter ( $\mathrm{km}$; gray lines represent 3-km contours), and (c) azimuthal shear $\left(10^{-3} \mathrm{~s}^{-1}\right.$, approximately one-half of the vertical vorticity; gray lines represent 15 and $20 \times 10^{-3} \mathrm{~s}^{-1}$ contours) for the tornado's parent mesocyclone. A distance scale (km) relative to Guangzhou radar and the local time (LST) are indicated on the horizontal axis. The strength of the TVS, defined by the gate-by-gate radial velocity difference, is indicated by circles in (a). Colors represent the elevation angles of the PPI scan associated with each quantity. The presence of the tornado, through visual and damage-track records, is indicated by the red line. 
outbound and inbound Doppler velocity maxima, respectively (Fig. 4). A mesocyclone usually possesses a diameter between 2 and $10 \mathrm{~km}$ and a vertical vorticity on the order of $0.01 \mathrm{~s}^{-1}$ or greater (American Meteorological Society 2016). Figure 5 illustrates the characteristics of the mesocyclone from the estimated axisymmetric rotational velocity, $V_{\text {rot }}=\left(V_{\max }-V_{\min }\right) / 2$, and the mesocyclone diameter $D$ defined as the distance between $V_{\text {max }}$ and $V_{\text {min }}$ (Lee and White 1998; Stumpf et al. 1998), and azimuthal shear (one-half of the vorticity), $\left(V_{\max }-V_{\min }\right) / D$. The cyclonic vortex associated with the tornado-producing miniature supercell (Fig. 5) easily met the criteria of a mesocyclone (Andra 1997; Lee and White 1998), with a vorticity exceeding $10^{-2} \mathrm{~s}^{-1}$ at 1430 LST and $\sim 3 \mathrm{~km}$ above ground level (AGL; hereafter all heights are AGL). The mesocyclone rapidly intensified below 3-km altitude, with $V_{\text {rot }} \sim 30 \mathrm{~m} \mathrm{~s}^{-1}$ at 1530 LST. It can be categorized as a strong mesocyclone according to the mesocyclone strength nomogram (Andra 1997). Associated with the strengthening of the mesocyclone, its diameter contracted from $\sim 6 \mathrm{~km}$ to less than $3 \mathrm{~km}$ at 1530 LST when the tornado was observed with vorticity exceeding $3 \times 10^{-2} \mathrm{~s}^{-1}$. The mesocyclone continued to contract toward $D \sim 2 \mathrm{~km}$ at 1542 LST while $V_{\text {rot }}$ peaked at $\sim 1530 \mathrm{LST}$ with vorticity exceeding $4.5 \times 10^{-2} \mathrm{~s}^{-1}$. This could be a sign of the mesocyclone intensifying and collapsing into a tornado vortex, as documented in Wakimoto et al. (1998). However, the Guangzhou radar's beamwidth $(\sim 400 \mathrm{~m})$ was too coarse to resolve the tornado $(D \sim 200 \mathrm{~m})$ at a distance of $\sim 25 \mathrm{~km}$ from the radar. It is noted that the lowest elevation of Guangzhou radar was $0.5^{\circ}$ such that the lowest level of the mesocyclone was better sampled as it moved closer to the radar from 1436 to 1546 LST. The EF3 tornado damage coincided with the time of peak mesocyclone intensity, consistent with previous studies that $~ 90 \%$ of EF3-EF5 tornadoes were associated with strong mesocyclones (e.g., Smith 1965). The mesocyclone weakened and broadened after 1536 LST (Fig. 5).

TVS. A TVS is defined as a velocity signature with a local maximum and minimum over an azimuthal distance of approximately one beamwidth $(<1 \mathrm{~km})$ and with gate-to-gate azimuthal radial velocity difference $\Delta V$ greater than $20 \mathrm{~m} \mathrm{~s}^{-1}$ (Brown et al. 1978). The TVS was first detected at $1526 \mathrm{LST}$ at the $2.4^{\circ}$ elevation angle (about $1.2 \mathrm{~km}$ ) near LD. By $1530 \mathrm{LST}$, the TVS intensified rapidly and descended toward the surface (Fig. 4b). It could be detected at all elevation angles below $9.9^{\circ}$ except $0.5^{\circ}$ (Fig. 5). The lack of a consistent
TVS at $0.5^{\circ}$ can be attributed to the strong second-trip echo contaminating the radial velocities in the TVS region. In fact, the first surface damage was identified near LD 1528 LST, and a video confirms the tornado was present at approximately 1530 LST. From 1530 to 1536 LST, the TVS at all elevations below $3.4^{\circ}$ continued to intensify with $\Delta V$ greater than $50 \mathrm{~m} \mathrm{~s}^{-1}$. The strongest TVS was detected from a planned position indicator (PPI) scan at the $0.5^{\circ}$ elevation angle at 1536 LST with a magnitude of $\Delta V \sim 60 \mathrm{~m} \mathrm{~s}^{-1}$; this was collocated with the severe damage exemplified by the demolished factories near LY. In the next $6 \mathrm{~min}$, the low-level TVS intensity decreased below $55 \mathrm{~m} \mathrm{~s}^{-1}$. The extreme radar reflectivity $(>60 \mathrm{dBZ})$ in the hook echo at 1536 and 1542 LST also is suggestive of a tornado debris signature (Figs. 4c,d). The strongest damage occurred at MD 1540 LST when a concrete pole with a diameter of $0.5 \mathrm{~m}$ was snapped (Fig. $3 \mathrm{~d}$ and blue dot in Fig. 5), supporting an EF3 tornado. After that, the TVS continued to weaken, coincident with the weak damages between JB and QK. From 1600 LST onward, the TVS became undetectable in radar data, consistent with the end of the damage pattern north of QK.

\section{KINEMATIC STRUCTURE OF THE MIN- IATURE SUPERCELL. Doppler radial velocities} from Guangzhou and Shenzhen radars were first interpolated onto a $1 \mathrm{~km} \times 1 \mathrm{~km} \times 1 \mathrm{~km}$ Cartesian grid using the National Center for Atmospheric Research's "REORDER" software (Oye et al. 1995), then synthesized into 3D winds using Custom Editing and Display of Reduced Information in Cartesian space (CEDRIC) software (Mohr et al. 1986). The miniature supercell motion ( $21.7 \mathrm{~m} \mathrm{~s}^{-1}$ toward an azimuth of $330^{\circ}$ relative to true north) was accounted for in the interpolation procedure. Although the Guangzhou radar is closer to the tornado, the scale of the dual-Doppler syntheses is determined by the coarser sampling resolution from the Shenzhen radar $\sim 80 \mathrm{~km}$ from the tornado. As a result, the dual-Doppler syntheses can only resolve the parent mesocyclone and the miniature supercell. The evolution and intensification of the mesocyclone circulation at the southeast end of the miniature supercell are illustrated using the storm-relative wind vectors at 1.5 and $3.5 \mathrm{~km}$ (Fig. 6).

The storm-relative velocity field at $1.5 \mathrm{~km}$ is dominated by a closed cyclonic circulation (Fig. 6a). The midlevel inflow (Fig. 6d) came from the southwest and turned cyclonically toward the north after it entered the storm. The vorticity maximum is located 

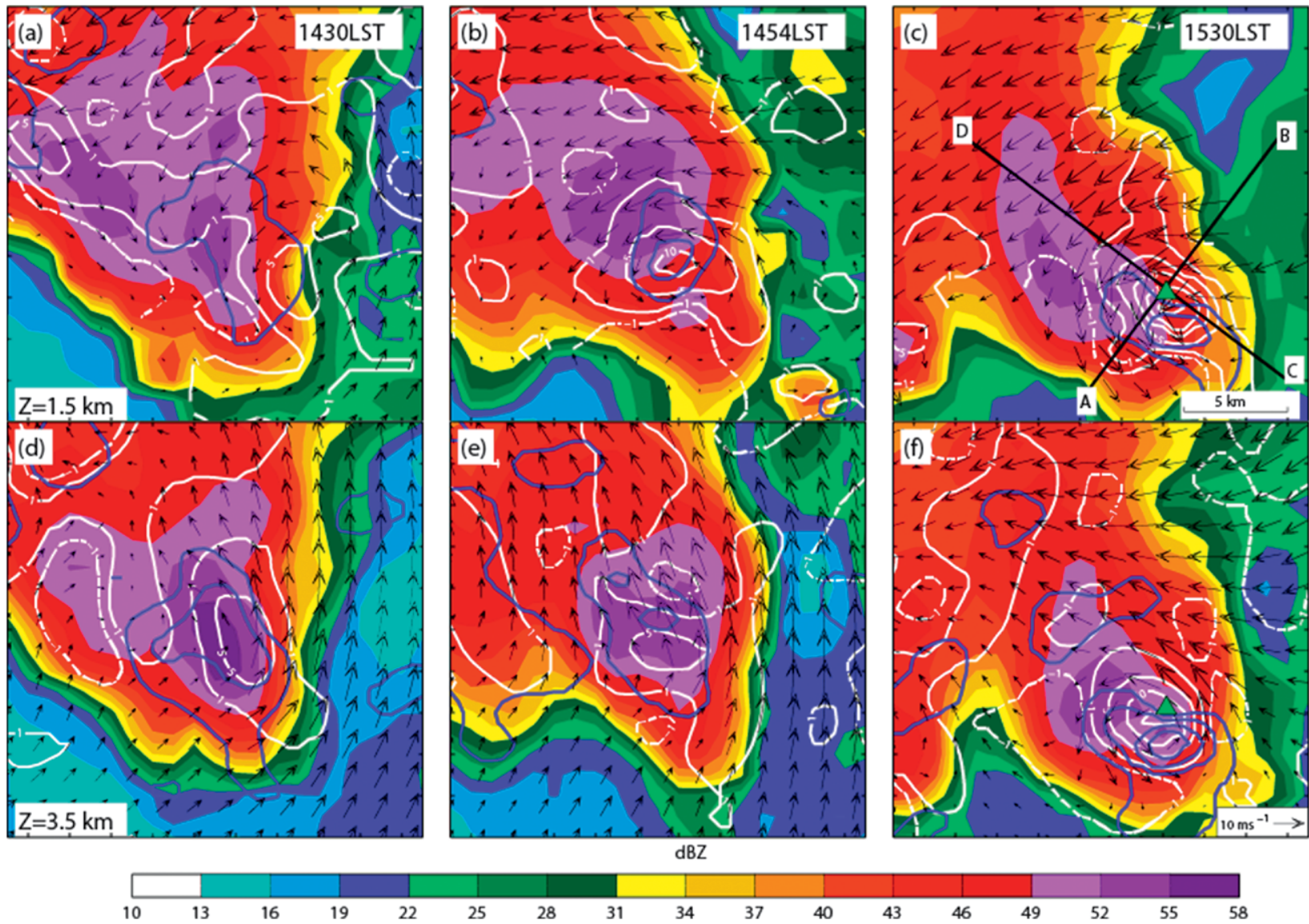

FIG. 6. Dual-Doppler analysis of tornadic mesocyclone from Guangzhou and Shenzhen radars at (a)-(c) I.5and (d)-(f) 3.5-km heights for before [(a),(d) 1430 and (b),(e) 1454 LST] and at the time of [(c),(f) 1530 LST] tornadogenesis. Reflectivity (color; $\mathrm{dBZ}$ ) is overlaid with storm-relative wind vectors. The positive (white solid contours) and negative (white dashed contours) vertical vorticity is shown at $\pm 1,5,10,15$, and $20 \times 10^{-3} \mathrm{~s}^{-1}$. The updraft (blue line) is contoured at $2,4,6,8$, and $10 \mathrm{~m} \mathrm{~s}^{-1}$. The green-filled triangles in (c) and (f) indicate the location of the TVS.

northwest of the low-level vorticity center, indicating tilting of the low-level horizontal vorticity aligned with the storm motion, consistent with the lowlevel shear vector as shown in Fig. 3. At 1454 LST (Figs. 6b,e), the mesocyclone at low levels intensified with its vorticity center collocated with the maximum updraft as the wind fields in the midlevels became more southerly. About 30 min later at 1530 LST (Figs. $6 c, f)$, the magnitude of the mesocyclone, accompanied by a hook echo, intensified by more than $100 \%$, with the vorticity maximum exceeding $2 \times 10^{-2} \mathrm{~s}^{-1}$ and its diameter decreased to less than $5 \mathrm{~km}$ (based on the $5 \times 10^{-3} \mathrm{~s}^{-1}$ vorticity contour). The tornado occurred near the center of the cyclonic circulation. Note that the vorticity maximum (i.e., the mesocyclone) is shifted north of the updraft center. The cyclonic circulation is well defined in the midlevels (Fig. 6f).
Figure 7 presents two orthogonal vertical cross sections of reflectivity, vertical velocity, and vertical vorticity through the mesocyclone center. The miniature supercell and its mesocyclone extend up to 8 and $4 \mathrm{~km}$ based on the $20-\mathrm{dBZ}$ and $5 \times 10^{-3} \mathrm{~s}^{-1}$ contours, respectively, with a maximum vorticity $\sim 2 \times 10^{-2} \mathrm{~s}^{-1}$ at $2 \mathrm{~km}$. These characteristics are consistent with miniature supercells observed in TC rainbands in the United States (e.g., Eastin et al. 2009; McCaul et al. 2004; Spratt et al. 1997). The storm-relative inflow approaches from the northeast, and the primary updraft extends from the boundary layer up to $\sim 6 \mathrm{~km}$ with a maximum of $\sim 10 \mathrm{~m} \mathrm{~s}^{-1}$ near $3-\mathrm{km}$ altitude. A moderate downdraft with a maximum of $\sim 6 \mathrm{~m} \mathrm{~s}^{-1}$ is confined to the north of the updraft within the inflow region, which can be attributed to the compensating downdraft of the convective updraft and the drag by 

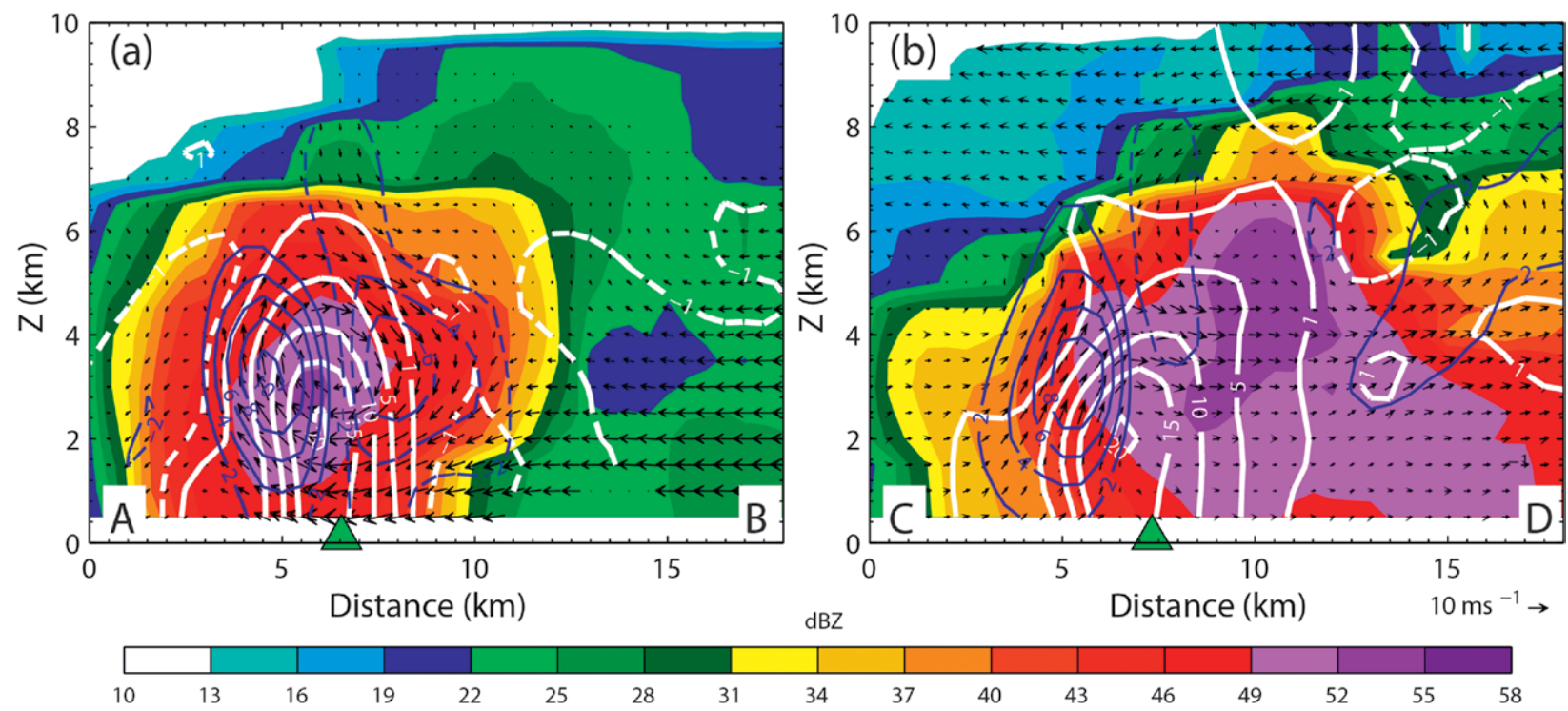

FIG. 7. Vertical cross sections of radar reflectivity (dBZ; color shading), storm-relative wind vectors, vertical velocity ( $\mathrm{m} \mathrm{s}^{-1}$; blue contours), and vertical vorticity $\left(I \times 10^{-3} \mathrm{~s}^{-1}\right.$; white contours) at I530 LST (at the time of tornadogenesis) along line segments (a) AB and (b) CD in Fig. 6c. The solid and dashed lines denote positive and negative values, respectively. The green-filled triangles indicate the location of TVS.

heavy precipitation $(>45 \mathrm{dBZ})$. The tornado is located at the boundary between the updraft and downdraft.

To examine the intensification mechanism of the mesocyclone in this miniature supercell, the vertical vorticity budget from the 3D dual-Doppler winds is calculated using the method described in Eastin et al. (2009). Ignoring contributions from frictional and solenoidal terms that are usually much smaller, the time rate of change of vertical vorticity can be expressed as the sum of horizontal (HADV) and vertical (VADV) advection of vertical vorticity, the convergence (CONV, or stretching) and tilting (TILT) terms. Figure 8 shows the horizontal distribution of the vertical vorticity production terms at height $Z=1.5 \mathrm{~km}$ in the vicinity of the mesocyclone at 1454 and 1530 LST, corresponding to the times before and near tornadogenesis, respectively. At 1454 LST (Figs. 8a-d), the CONV term is mostly positive within the mesocyclone. The tilting term is comparable in magnitude to the CONV term and has positive contributions to the vorticity of the mesocyclone. Both the HADV and the VADV terms exhibit a pair of positive and negative maxima near the center of the mesocyclone, consistent with the effect of advection by the storm-relative winds and vertical motion. Overall, the low-level vorticity production before tornadogenesis mostly resulted from tilting of horizontal vorticity and its subsequent stretching.

Near the time of tornadogenesis ( 1530 LST; Figs. 7e-h), the CONV and HADV terms increased to an order of magnitude larger than VADV and TILT. The HADV term still exhibits a positive (negative) maximum at the southwest (northeast) flank of the mesocyclone center. Maximum CONV exceeds $25 \times 10^{-6} \mathrm{~s}^{-2}$ and is collocated with the mesocyclone, indicating its dominant role in the production of vertical vorticity. The vorticity distribution and evolution in this event are consistent with supercells found in the U.S. Great Plains, where the vertical vorticity couplet is initially created by tilting of horizontal environmental vorticity, and then positive vorticity is rapidly intensified by the stretching of vertical vorticity within the mesocyclone (e.g., Davies-Jones et al. 2001; McCaul and Weisman 1996).

Past studies (e.g., Dawson et al. 2010; Markowski et al. 2002) have pointed to the importance of the cold pool, including its strength, in tornadogenesis. For the miniature supercell storm studied here, the cold pool was rather weak; the surface temperature drops were less than $1 \mathrm{~K}$ at surface stations near the path of the tornado (not shown), which is not surprising because of the very humid low-level environment. Hence, the baroclinically generated vorticity should not be an important factor.

CONCLUDING REMARKS. The first look at the evolution and structure of a miniature supercell in Typhoon Mujigae (2015), which spawned an EF3 tornado, the strongest tornado embedded in a typhoon rainband ever observed in China, was presented in this paper 

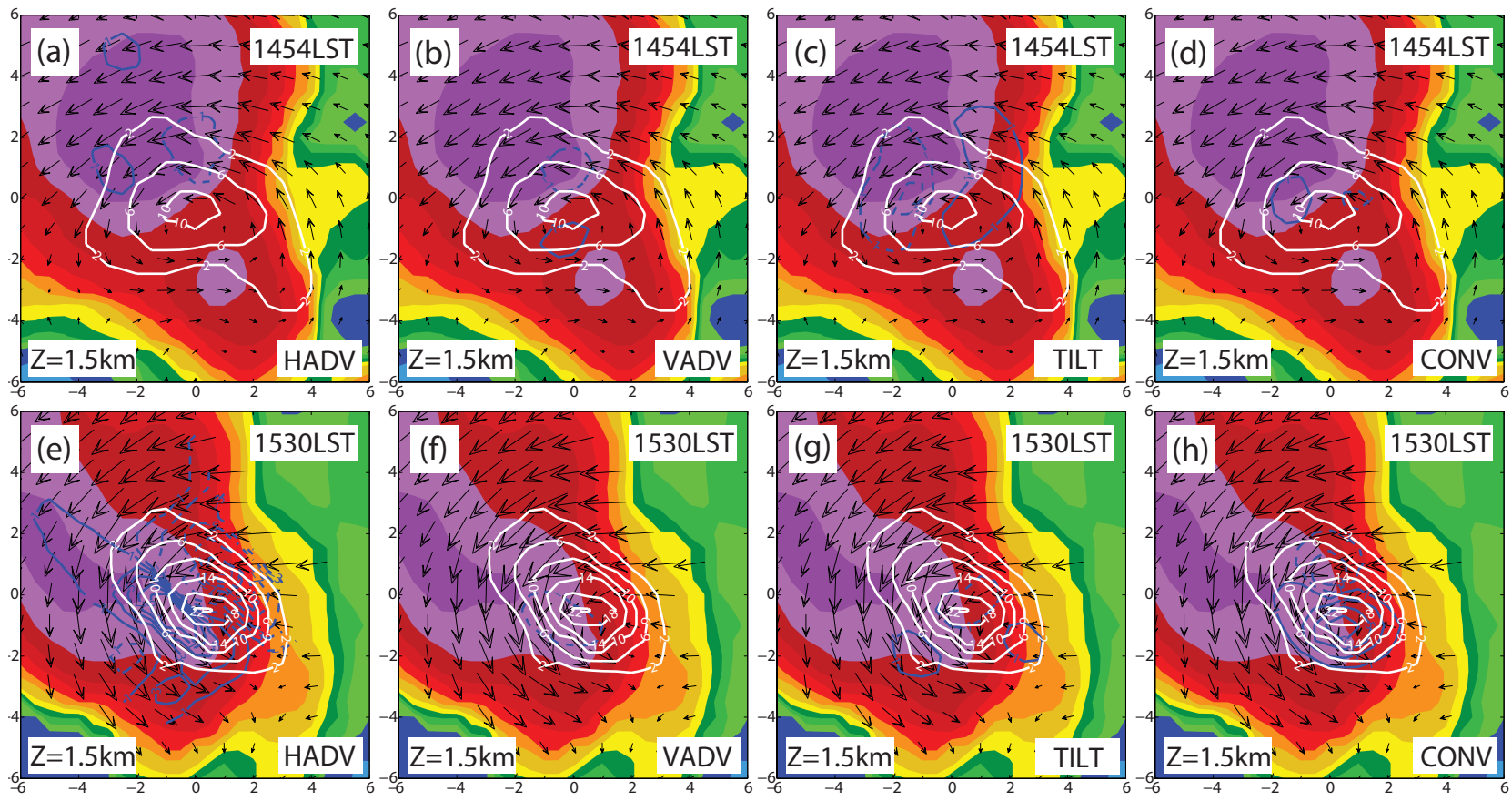

\begin{tabular}{ll|lllllllllllllllll}
\hline & $\frac{1}{10}$ & 13 & 16 & 19 & 22 & 25 & 28 & 31 & 34 & 37 & 40 & 43 & 46 & 49 & 52 & 55 & 58 & $\mathrm{dBZ}$
\end{tabular}

FIG. 8. Distributions of (a),(e) HADV; (b),(f) VADV; (c),(g) TILT; and (d),(h) CONV terms within the mesocyclone at $1.5 \mathrm{~km}$ at (a)-(d) 1454 and (e)-(h) 1530 LST, corresponding to the times before and near tornadogenesis, respectively. Blue solid (dashed) contours denote positive (negative) contributions to vertical vorticity production, with an interval of $2 \times 10^{-6} \mathrm{~s}^{-2}$. Reflectivity (dBZ; color shading) is overlaid with storm-relative wind vectors and vertical relative vorticity (white contours; interval of $4 \times 10^{-3} \mathrm{~s}^{-1}$ ).

using single- and dual-Doppler radar data along with rawinsonde and damage surveys. The tornadic minisupercell was embedded in an environment of moderate CAPE, strong low-level shear, a low lifting condensation level, and a small surface dewpoint depression, similar to its U.S. hurricane counterparts. Mesocyclone properties deduced from single-Doppler radar data suggest that the mesocyclone first intensified and contracted at $\sim 3-\mathrm{km}$ altitude and then descended to lower levels. Because of the limitations of the available data, we are unable to investigate the details of tornadogenesis and the subsequent evolution and structure of the tornado.

With dual-Doppler wind analyses, the parent mesocyclone was well identified. Positive vorticity intensified as the hook-echo reflectivity signature developed. The vorticity budget demonstrated that tilting of the low-level horizontal vorticity into the vertical and subsequent stretching by a strong updraft were the main contributors to the mesocyclone intensification. Future studies will examine the microphysical processes of the miniature supercell using dual-polarization data from the Hong Kong radar. In addition, storm dynamics and tornadogenesis will be examined using high-resolution numerical simulations that include radar data assimilation.

ACKNOWLEDGMENTS. This work was primarily supported by the National Fundamental Research 973 Program of China (Grants 2013CB430101 and 2015CB452801), the National Natural Science Foundation of China (Grants 41322032, 41275031 and 41230421), and the Social Commonwealth Research Program (Grant GYHY201006007). We would like to acknowledge the China Meteorological Administration for organizing damage surveys and collecting the radar data used in this study. Special thanks also go to everyone who took part in the tornado damage survey and shared their valuable pictures and videos of the tornado damage. We would also like to acknowledge Huang Sheng, who shared his useful videos of the tornado.

\section{FOR FURTHER READING}

American Meteorological Society, 2016: Glossary of Meteorology. 2nd ed. 850 pp. [Available online at http:// glossary.ametsoc.org/wiki/Mesocyclone.] 
Andra, D. L., 1997: The origin and evolution of the WSR-88D mesocyclone recognition nomogram. Preprints, 28th Conf. Radar Meteorology, Austin, TX, AMS, 364-365.

Brown, R. A., L. R. Lemon, and D. W. Burgess, 1978: Tornado detection by pulsed Doppler radar. Mon. Wea. Rev., 106, 29-38, doi:10.1175/1520 -0493(1978)106<0029:TDBPDR>2.0.CO;2.

Davies-Jones, R., R. J. Trapp, and H. B. Bluestein, 2001: Tornadoes and tornadic storms. Severe Convective Storms, C. A. Doswell III, Ed., Springer, 167-221.

Dawson, D. T., M. Xue, J. A. Milbrandt, and M. Yau, 2010: Comparison of evaporation and cold pool development between single-moment and multimoment bulk microphysics schemes in idealized simulations of tornadic thunderstorms. Mon. Wea. Rev., 138, 1152-1171, doi:10.1175/2009MWR2956.1.

Eastin, M. D, and Link, M. C., 2009: Miniature supercells in an offshore outer rainband of Hurricane Ivan (2004). Mon. Wea. Rev., 137, 2081-2104, doi:10.1175/2009MWR2753.1.

Edwards, R., 2012: Tropical cyclone tornadoes: A review of knowledge in research and prediction. Electronic J. Severe Storms Meteor., 7., 1-61.

Fan, W., and X. Yu, 2015: Characteristics of spatialtemporal distribution of tornadoes in China. Meteorology, 41, 793-805 (in Chinese).

Green, B. W., F. Zhang, and P. Markowski, 2011: Multiscale processes leading to supercells in the landfalling outer rainbands of Hurricane Katrina (2005). Wea. Forecasting, 26, 828-847, doi:10.1175 /WAF-D-10-05049.1.

Lee, R. R., and A. White, 1998: Improvement of the WSR88D mesocyclone algorithm. Wea. Forecasting, 13, 341-351, doi:10.1175/1520-0434(1998)013<0341:IO TWMA $>2.0 . C O ; 2$.

Lee, W. C., M. M. Bell, and K. E. Goodman, 2008: Supercells and mesocyclones in outer rainbands of Hurricane Katrina (2005). Geophys. Res. Lett., 35, L16803, doi:10.1029/2008GL034724.

Markowski, P. M., J. M. Straka, and E. N. Rasmussen, 2002: Direct surface thermodynamic observations within the rear-flank downdrafts of nontornadic and tornadic supercells. Mon. Wea. Rev., 130, 1692-1721, doi:10.1175/1520-0493(2002)130<1692:DSTOWT $>2.0 . \mathrm{CO} ; 2$.

McCaul, E. W., Jr., 1987: Observations of the Hurricane "Danny" tornado outbreak of 16 August 1985. Mon. Wea. Rev., 115, 1206-1223, doi:10.1175/1520 -0493(1987)115<1206:OOTHTO>2.0.CO;2.
_ 1991: Buoyancy and shear characteristics of hurricane-tornado environments. Mon. Wea. Rev., 119, 1954-1978, doi:10.1175/1520-0493(1991)119<1954:BA $\mathrm{SCOH}>2.0 . \mathrm{CO} ; 2$.

— supercell storms in landfalling hurricane environments. Mon. Wea. Rev., 124, 408-429, doi:10.1175/1520 -0493(1996)124<0408:SOSSSI>2.0.CO;2.

- D. E. Buechler, S. J. Goodman, and M. Cammarata, 2004: Doppler radar and lightning network observations of a severe outbreak of tropical cyclone tornadoes. Mon. Wea. Rev., 132, 1747-1763, doi:10.1175/1520 -0493(2004)132<1747:DRALNO>2.0.CO;2.

McDonald, J. R., and K. C. Mehta, 2006: A recommendation for an Enhanced Fujita scale (EF-Scale). Wind Science and Engineering Center, Texas Tech University.

Meng, Z., and D. Yao, 2014: Damage survey, radar, and environment analyses on the first-ever documented tornado in Beijing during the heavy rainfall event of 21 July 2012. Wea. Forecasting, 29, 702-724, doi:10.1175/WAF-D-13-00052.1.

Mohr, C. G., L. J. Miller, R. L. Vaughan, and H. W. Frank, 1986: The merger of mesoscale datasets into a common Cartesian format for efficient and systematic analyses. J. Atmos. Oceanic Technol., 3, 143-161, doi:10.1175/1520-0426(1986)003<0143:TMOMDI $>2.0 . \mathrm{CO} ; 2$.

Oye, R., C. Mueller, and S. Smith, 1995: Software for radar translation, visualization, editing, and interpolation. Preprints, 27th Conf. Radar Meteorology, Vail, CO, AMS, 359-361.

Saito, R., M. Fujita, G. Dresselhaus, and M. S. Dresselhaus, 1992: Electronic structure of chiral graphene tubules. Appl. Phys. Lett., 60, 2204-2206, doi:10.1063/1.107080.

Schneider, D., and S. Sharp, 2007: Radar signatures of tropical cyclone tornadoes in central North Carolina. Wea. Forecasting, 22, 278-286, doi:10.1175 /WAF992.1.

Schultz, L. A., and D. J. Cecil, 2009: Tropical cyclone tornadoes, 1950-2007. Mon. Wea. Rev., 137, 3471-3484, doi:10.1175/2009MWR2896.1.

Smith, J. S., 1965: The hurricane-tornado. Mon. Wea. Rev., 93, 453-459, doi:10.1175/1520-0493(1965)093 $<0453:$ THT $>2.3$.CO;2.

Spratt, S. M., D. W. Sharp, P. Welsh, A. Sandrik, F. Alsheimer, and C. Paxton, 1997: A WSR-88D assessment of tropical cyclone outer rainband tornadoes. Wea. Forecasting, 12, 479-501, doi:10.1175/1520 -0434(1997)012<0479:AWAOTC>2.0.CO;2. 
Stumpf, G. J., and Coauthors, 1998: The National Severe Storms Laboratory Mesocyclone Detection Algorithm for the WSR-88D. Wea. Forecasting, 13, 304-326, doi:10.1175/1520-0434(1998)013<0304:TN SSLM>2.0.CO;2.

Suzuki, O., H. Niino, H. Ohno, and H. Nirasawa, 2000: Tornado-producing mini supercells associated with Typhoon 9019. Mon. Wea. Rev., 128, 1868-1882, doi:10.1175/1520-0493(2000)128<1868:TPMSAW > 2.0.CO;2

Wakimoto, R. M., C. Liu, and H. Cai, 1998: The Garden City, Kansas, storm during VORTEX 95. Part I: Overview of the storm's life cycle and mesocyclogenesis. Mon. Wea. Rev., 126, 372-392, doi:10.1175/1520 -0493(1998)126<0372:TGCKSD>2.0.CO;2.
Xue, M., K. Zhao, M. Wang, Z. Li, and Y. Zheng, 2016: Recent significant tornadoes in China. $A d v$. Atmos. Sci., 33, 1209-1217, doi:10.1007/s00376016-6005-2.

Yu, H., P. Chen, Q. Li, and B. Tang, 2013: Current capability of operational numerical models in predicting tropical cyclone intensity in the western North Pacific. Wea. Forecasting, 28, 353-367, doi:10.1175 /WAF-D-11-00100.1.

Zheng, Y., B. Zhang, X. Wang, K. Sun, R. Mu, and W. Xia, 2015: Analysis of typhoon-tornado weather background and radar echo structure. Meteor. Mon., 41, 942-952 (in Chinese with English abstract).

\section{NEW FROM AMS BOOKS!}

\section{Weather in the Courtroom}

Memoirs from a Career in

Forensic Meteorology

\section{William H. Haggard}

From a pioneering forensic meteorologist...

The inside scoop on legendary litigations where the weather may — or may not-have played a crucial role, including:

- The disappearance of an Alaskan congressman's airplane in 1972

- The collapse of Tampa Bay's Skyway Bridge in 1980

- The crash of Delta Flight 19I in Dallas/Fort Worth in 1985

William H. Haggard is former director of the NCDC and AMS certified consulting meteorologist (CCM), fellow, and honorary member.

(C) 2016, paperback, 240 pages

ISBN 13: 978-I-940033-95-2

List price: $\$ 30 \quad$ AMS Member price: $\$ 20$
WEATHER IN THE

\section{COURTROOM}

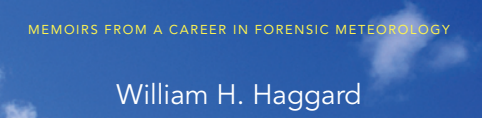

William H. Haggard

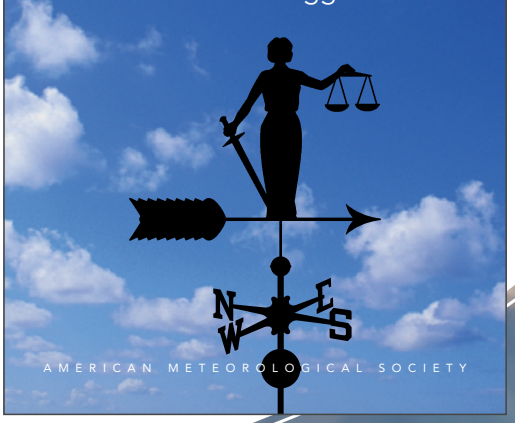

AMS BOOKS RESERRCH MPPLCATIONS ' HISTORY bookstore.ametsoc.org 


\section{LOOKING FOR AN EXPERT?}

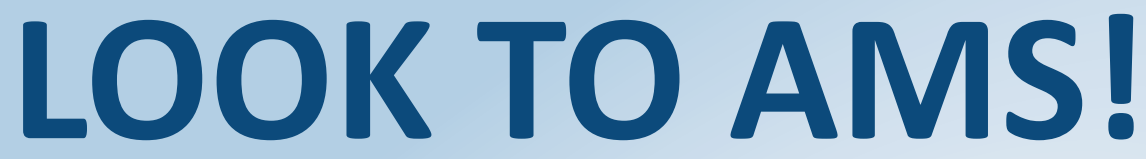

\section{AMS announces the launch of our new online directory of Weather and Climate Service Providers.}

This new online directory replaces the former BAMS Professional Directory and lists an array of weather and climate service providers. You can find the new directory under the "Find an Expert" link from the AMS home page.

It's easier than ever for the weather, water, and climate community and the general public to search for organizations and individuals offering these important services.

\section{Learn more at www.ametsoc.org}
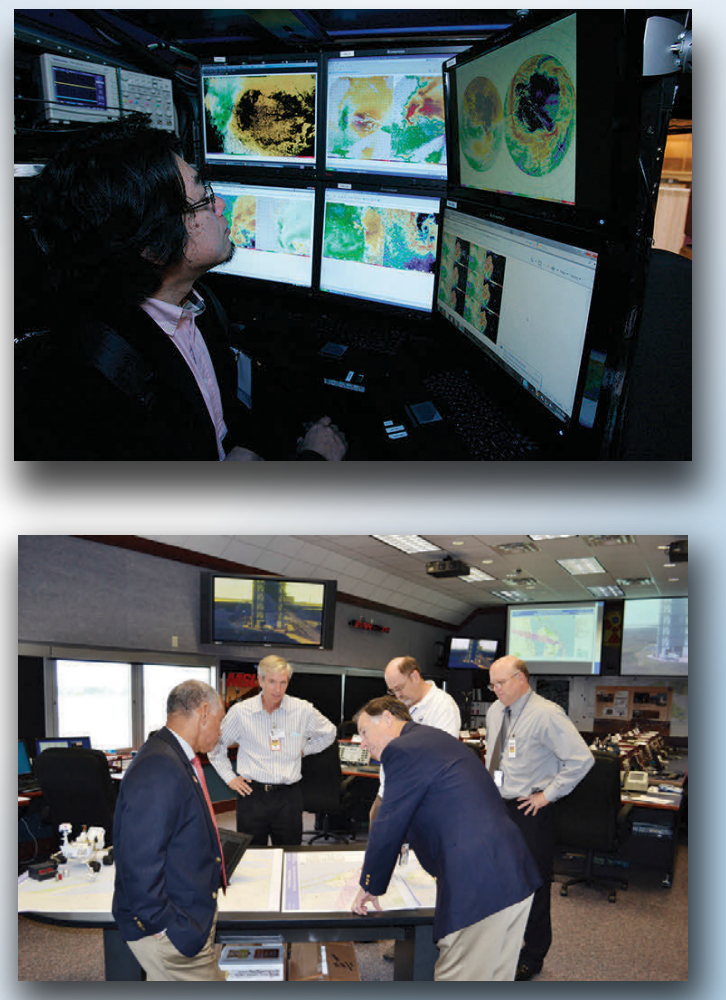

\section{NEW!}

\section{Weather \& Climaite Service Providers Directory}

